\title{
Comparison of Nucleoside Triphosphate Levels in the Wild-type Strain with Those in Sporulation-deficient and Solvent-deficient Mutants of Clostridium acetobutylicum P262
}

\author{
By J. D. SANTANGELO, D. T. JONES AND D. R. WOODS* \\ Department of Microbiology, University of Cape Town, Rondebosch 7700, South Africa
}

(Received 2 June 1988; revised 13 October 1988; accepted 7 November 1988)

\begin{abstract}
The role of GTP and other ribonucleoside phosphates in the differentiation of Clostridium acetobutylicum and two sporulation-deficient mutants was investigated. The clostridial stage $(\mathrm{cls}-1)$ mutant was unable to produce the clostridial stage, solvents, granulose, capsules or endospores, whereas the sporulation-deficient (spo-1) mutant produced the clostridial stage, solvents, granulose and capsules, but did not form a forespore septum. The nucleoside triphosphate profiles of the wild-type and spo-1 mutant were similar and were characterized by a trough in nucleotide levels which occurred just prior to the $\mathrm{pH}$ break point, the onset of the stationary growth phase, clostridial stage formation and the transition from the acidogenic to the solventogenic phase. The nucleoside triphosphate concentrations during the exponential growth phase were much lower than those found during the stationary phase. The nucleotide levels in the $c l s-1$ mutant during the exponential phase were comparable to those observed in the wild-type and spo-1 mutant. However, the nucleotide levels in the $c l s-1$ mutant did not increase during the stationary growth phase. The involvement of nucleotide levels, particularly that of GTP, in the differentiation of C. acetobutylicum was indicated by the effect of inhibitors, which have been shown to decrease ribonucleotide levels in other organisms and cause an increase in sporulation.
\end{abstract}

\section{INTRODUCTION}

The obligate anaerobe Clostridium acetobutylicum is used for the production of acetone, butanol and ethanol (ABE). In some strains of $C$. acetobutylicum, such as the industrial strain $\mathrm{P} 262$, the onset of solvent production and sporulation is characterized by a change in morphology from a highly motile, acid-producing, vegetative rod to a swollen, granulosecontaining, phase-bright, cigar-shaped, solvent-producing, 'clostridial' cell (Long et al., 1983; Jones \& Woods, 1986). An understanding of the triggers which lead to the onset of solvent production and differentiation is important for developments aimed at improving the $\mathrm{ABE}$ fermentation.

In Bacillus subtilis, a decrease in GTP content is associated with the initiation of sporulation (Lopez et al., 1979; Freese, 1981; Ochi \& Freese, 1983). A similar decrease in GTP or $S$-adenosylmethionine content was shown to be associated with differentiation (meiosis and sporulation) in Saccharomyces cerevisiae (Freese et al., 1984b). Recently, aerial mycelium formation in Streptomyces MA406-A-1 has been linked with a decrease in GTP content (Ochi, 1986). Since the role of GTP and other ribonucleoside phosphates in the differentiation of $C$. acetobutylicum has not been reported, we investigated changes in the ribonucleoside phosphate pools of the wild-type strain P262 and two sporulation-deficient mutants of this

Abbreviation: ABE fermentation, acetone/butanol/ethanol fermentation. 
strain. The clostridial stage $(\mathrm{cls}-\mathrm{l})$ mutant is unable to form the clostridial stage and can not produce solvents, capsule, granulose or endospores (Jones et al., 1982). The second mutant (spo-I) forms the clostridial stage and produces solvents, capsule and granulose but does not form a forespore septum (Jones et al., 1982).

\section{METHODS}

Bacterial strains. The wild-type C. acetobutylicum strain P262 has been described previously (Jones et al., 1982; Long et al., 1983). The $c l s-1$ and spo- 1 mutants were isolated from this strain by mutagenesis with ethyl methanesulphonate as described by Jones et al. (1982).

Media and culture conditions. The $C$. acetobutylicum strains were grown under stringent anaerobic conditions in the $C$, acetobutylicum minimal medium (CAMM) of Long et al. (1983), with the glucose concentration reduced from $60 \mathrm{~g} \mathrm{l}^{-1}$ to $50 \mathrm{~g} \mathrm{l}^{-1}$ and the nitrogen source changed from ammonium phosphate to ammonium nitrate $\left(7 \cdot 2 \mathrm{~g} \mathrm{l}^{-1}\right)$. CAMM was inoculated with exponential-phase cells $\left(\mathrm{OD}_{600} 0.45\right.$ to 0.50$)$ harvested from the clostridial basal medium (CBM) of O'Brien \& Morris (1971). All cultures were incubated at $34^{\circ} \mathrm{C}$ and shaken gently every $6 \mathrm{~h}$.

Morphology and end-product determinations. Total cell counts, clostridial stage counts, forespore counts and spore counts were determined with a Thoma counting chamber (Weber Scientific International) and a Zeiss photomicroscope fitted with phase- and interference-contrast optics. Acetate, butyrate, acetone, butanol and ethanol were determined by gas chromatography on a Hewlett Packard 5880A gas chromatograph fitted with a flame ionization detector, using 2-propanol as an internal standard. The column and running conditions have been described previously (Long et al., 1984).

Preparation of nucleotide extracts. Nucleotide extracts were prepared from cells grown in CAMM. The extraction methods of Olempska-Beer and Freese (1984) and Payne \& Ames (1982) were combined and modified for use with $C$. acetobutylicum. Cells $(100 \mathrm{ml})$ were collected rapidly on a $100 \mathrm{~mm}$ diameter nitrocellulose filter (Schleicher and Schüll BA 85 or AE 95) by vacuum filtration. The filter was placed cell side down in a glass Petri dish containing $5 \mathrm{ml}$ ice-cold $3 \mathrm{M}$-formic acid saturated with 1-butanol. After a $30 \mathrm{~min}$ extraction period, the liquid was removed and the filter and Petri dish were washed twice with ice-cold sterile water. The three liquid fractions were combined and adjusted to $\mathrm{pH} 8.8$ with $25 \%(\mathrm{w} / \mathrm{v})$ ammonia solution. The salt concentration of the extract was increased to $1 \mathrm{~m}$ by the addition of $3 \mathrm{M}$-ammonium acetate, $\mathrm{pH} 8 \cdot 8$. Debris was removed by centrifugation at $40000 \mathrm{~g}$ for $20 \mathrm{~min}$. The supernatant was applied to a column $(1.5 \mathrm{~cm}$ long $\times 1 \mathrm{~cm}$ diameter $)$ packed with Affi-Gel 601 (Bio-Rad) which had been equilibrated with $1 \mathrm{M}$-ammonium acetate, $\mathrm{pH} 8 \cdot 8$. The absorbance of the effluent was monitored at $254 \mathrm{~nm}$ and unwanted and interfering components were washed through the column with a mobile phase of $1 \mathrm{M}$-ammonium acetate, $\mathrm{pH} 8.8$. When the $A_{254}$ of the effluent reached baseline, the mobile phase was changed to $100 \mathrm{~mm}$-acetic acid to initiate elution of the nucleotides. The desired sample which contained the nucleotides (about $10 \mathrm{ml}$ ) was collected and freeze-dried. The freeze-dried sample was resuspended in water, centrifuged and assayed by high-performance liquid chromatography (HPLC). The reliability of this extraction method was routinely tested by performing duplicate extractions where a known concentration of a standard nucleotide mixture was added to one sample just prior to the formic acid treatment (Freese et al., 1984a).

The nucleotide extraction method of Olempska-Beer \& Freese (1984) utilized $1 \mathrm{M}$-formic acid saturated with 1-butanol. In our system, the concentration of formic acid had to be increased to $3 \mathrm{M}$ to overcome the high buffering capacity of CAMM, which contained calcium carbonate. This was consistent with the results of Bagnara \& Finch (1972), who reported that formic acid was unsatisfactory for the extraction of nucleotides if the buffering capacity of the culture medium caused the $\mathrm{pH}$ of the extractant to rise above approximately $3 \cdot 0$. The formic acid extract had to be purified prior to the HPLC analysis. Similar purification procedures were also reported as an essential step for the analysis of nucleotides from plant tissues (Meyer \& Wagner, 1985; Payne \& Ames, 1982).

Analysis of nucleotide extracts. The nucleotides in the cell extracts were separated using strong anion-exchange HPLC. The computer-HPLC data collection and analysis system used to analyse the nucleotide composition of the extracts has been described previously (Santangelo, 1988). All experiments were done at least three times, with similar results; representative data are presented.

Nucleotide synthesis inhibitors. Decoyinine and psicofuranine were generously donated by Dr R. L. Keen, Upjohn Company, Kalamazoo, Michigan, USA. Caffeine and 3-methylxanthine were purchased from Fluka. Theobromine, theophylline, 6-mercaptopurine, 2-amino-6-mercaptopurine and 6-azathymine were purchased from Sigma. Since CAMM supports a near-maximum spore titre for $C$. acetobutylicum $\mathbf{P} 262$, it was an unsuitable medium for inhibitor studies where an increase in sporulation was to be determined. The tryptone/yeast extract/glucose medium (TYG) of Hongo et al. (1968), which resulted in a lower sporulation level, was therefore used for these inhibitor studies. The inhibitors were added during the mid-exponential growth phase, when the $\mathrm{OD}_{600}$ of the culture was $0 \cdot 40$ to $0 \cdot 45$. Percentage sporulation was determined after $48 \mathrm{~h}$. 


\section{RESULTS}

Growth, physiology and morphology

The ABE fermentation profile typical of $C$. acetobutylicum P262 in CAMM is shown in Fig. $1(a, b)$. Cells transferred to this medium show a characteristic $15 \mathrm{~h}$ lag phase after which cell numbers begin to increase (Long et al., 1983). The exponential growth phase, from 15 to $35 \mathrm{~h}$, was characterized by motile, rod-shaped cells. During this stage, glucose consumption resulted in acid production. The $\mathrm{pH}$ break point occurred when the acid concentration reached a maximum of approximately $4 \mathrm{~g} \mathrm{l}^{-1}$ (between 30 and $35 \mathrm{~h}$ ). The break point was associated with the onset of the stationary growth phase and clostridial stage formation. Just prior to the $\mathrm{pH}$ break point, the cell morphology began to change from motile rods to phase-bright, granulosefilled clostridial cells, and by $40 \mathrm{~h} 90$ to $100 \%$ of the cells had converted to the clostridial stage. The physiological changes associated with the $\mathrm{pH}$ break point included a decrease in acid concentration, initiation of solvent production, and an increase in the rate of glucose consumption. After $45 \mathrm{~h}$, the acid concentration stabilized at approximately $2 \cdot 7 \mathrm{~g} \mathrm{l}^{-1}$. Glucose exhaustion occurred at approximately $50 \mathrm{~h}$ and shortly thereafter solvent levels reached a peak of approximately $10 \mathrm{~g} \mathrm{l}^{-1}$. At this time a maximum number of cells containing forespore septa $(60 \%)$ was observed, as well as a small percentage of mature spores $(15 \%)$. The highest proportion of phase-bright spores (approximately 50\%) was observed at the end of the fermentation $(67 \mathrm{~h})$.

A similar fermentation profile for the spo-1 mutant is shown in Fig. $1(c, d)$. The exponential phase was characterized by motile rods, glucose consumption and acid production. The $\mathrm{pH}$ break point ( $\mathrm{pH} \mathrm{5.3)} \mathrm{was} \mathrm{reached} \mathrm{between} 35$ and $40 \mathrm{~h}$ and was associated with a peak in acid levels. Stationary phase occurred after approximately $40 \mathrm{~h}$ and by $55 \mathrm{~h} 90$ to $100 \%$ of the cells were phase-bright clostridial forms. Solvents were first detected immediately after the $\mathrm{pH}$ break point and reached a maximum of $9.6 \mathrm{~g} \mathrm{l}^{-1}$ at the end of the fermentation $(67 \mathrm{~h})$. The major difference between this and the wild-type fermentation was that no forespore septa or mature spores were observed, indicating that the $s p o-1$ mutant was an early sporulation mutant $(s p o 0)$. Minor differences involving for example maximum cell number, time of peak in motility, and residual glucose levels were observed between the fermentation profiles of the P262 wild-type and spo- $l$ strains.

The $c l s-1$ mutant showed a normal motile, acid-producing, glucose-consuming exponential phase, but the switch to solvent production did not occur and no clostridial-stage cells were observed (Fig. $1 e, f$ ). After approximately $35 \mathrm{~h}$ the rate of glucose consumption slowed and acid levels continued to increase. By $55 \mathrm{~h}$ the fermentation ceased and virtually no solvents were produced $\left(<0.25 \mathrm{~g}^{-1}\right)$. Only $30 \%$ of the available glucose was utilized and approximately $7 \mathrm{~g}$ acids $1^{-1}$ were produced.

\section{Nucleotide profiles}

The nucleoside triphosphate profile for the wild-type $C$. acetobutylicum P262 strain demonstrated that throughcut the fermentation ATP was the most abundant nucleoside triphosphate followed by UTP, GTP and CTP (Fig. 2a). The nucleoside triphosphate concentrations during the exponential growth phase ( 15 to $35 \mathrm{~h}$ ) were much lower (by as much as 40-fold in the case of ATP) than the values obtained during most of the stationary growth phase $(40$ to $50 \mathrm{~h}$ ). From 17 to $23 \mathrm{~h}$ there was a gradual decrease in the concentration of all the nucleoside triphosphates, and just prior to the $\mathrm{pH}$ break point $(25$ to $35 \mathrm{~h})$ the concentration of all the nucleoside triphosphates decreased to almost zero $(27 \mathrm{~h})$. The nucleotide levels then increased rapidly as the morphology of the culture changed from phase-dark rods to phasebright clostridial cells. The highest pool values for all the nucleoside triphosphates coincided with the peak in the number of clostridial-stage cells and the onset of forespore septum development, indicating that the nucleoside triphosphate pool values were higher in the stationary-phase clostridial stage cells than in the exponential-phase rods. The decrease in the number of clostridial cells after $47 \mathrm{~h}$ correlated with a decrease in the nucleoside triphosphate concentrations, maximum solvent levels and the initiation of spore maturation. 

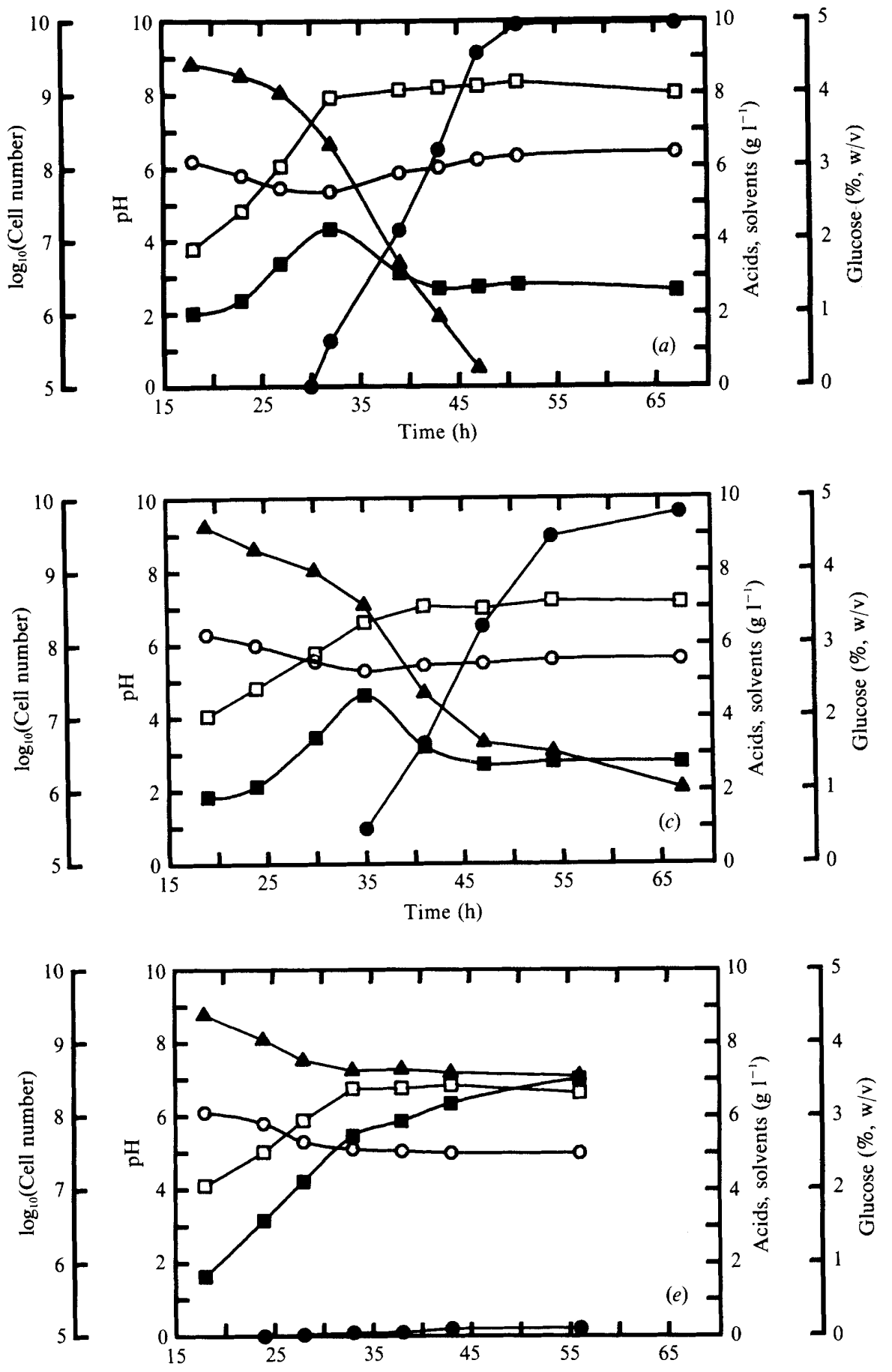

Fig. 1. Morphological and physiological profiles for $C$. acetobutylicum. ( $a, b$ ) Profiles for the wild-type strain $\mathrm{P} 262 ;(c, d)$ profiles for the spo- 1 mutant; $(e, f)$ profiles for the $c l s-1$ mutant. Symbols for graphs $(a),(c)$ and $(e)$ correspond to the following: $\mathbf{A}$, glucose concentration $(\%, \mathrm{w} / \mathrm{v}) ; \mathbf{\square}$, total acids $\left(\mathrm{g} \mathrm{l}^{-1}\right)$; , total solvents $\left(\mathrm{g} \mathrm{l}^{-1}\right) ; \square, \log$ cell number; $O, \mathrm{pH}$. The plots in $(b),(d)$ and $(f)$ (on facing page) are 

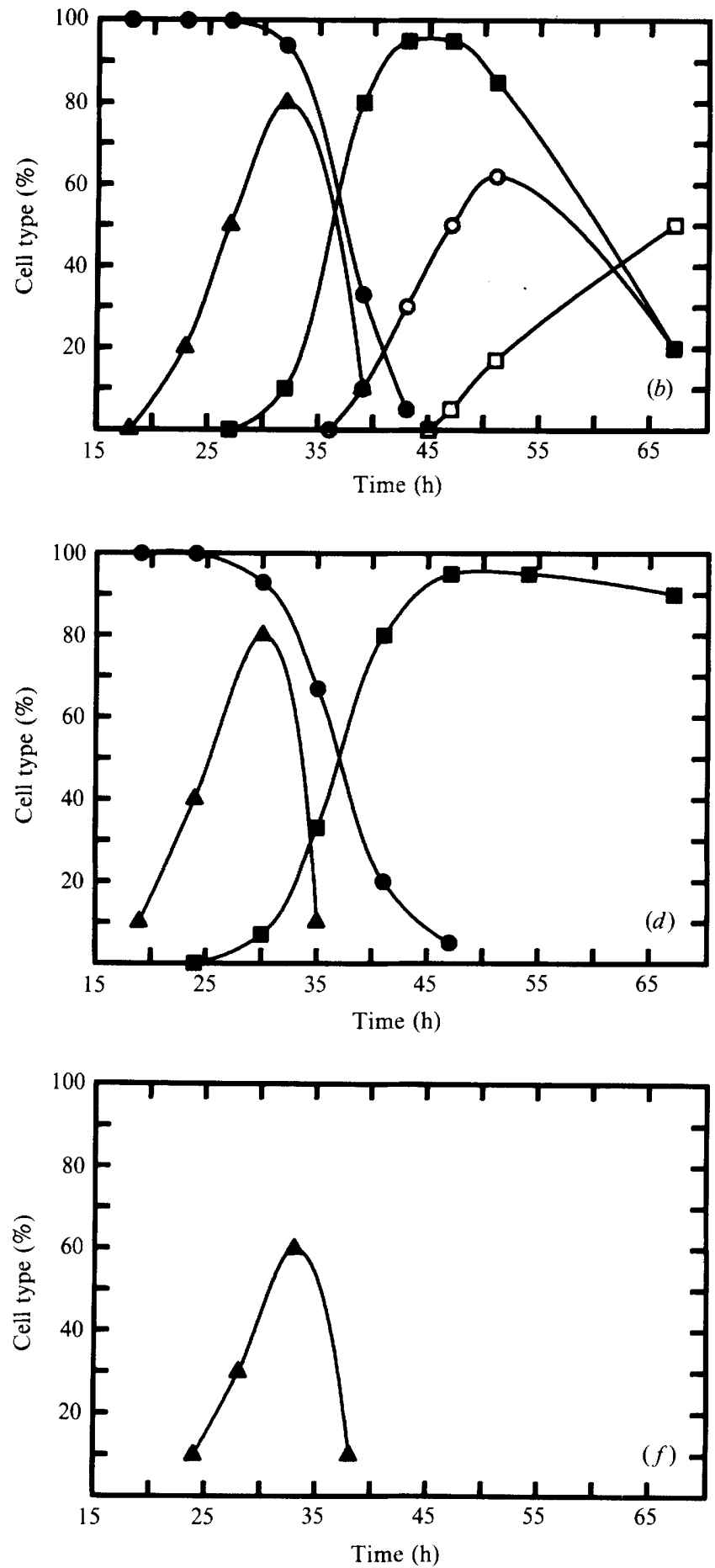

Fig. 1 (continued)

expressed as percentages of the total cell count, and the symbols correspond to the following: $\boldsymbol{\Delta}$, motility; $\boldsymbol{\square}$, phase-bright clostridial cells; $\boldsymbol{0}$, phase-dark rods; $\bigcirc$, forespore-containing cells; $\square$, mature spores. In $(f)$, only motility is shown; the $c l s-1$ mutant remained as $100 \%$ phase-dark rods throughout the fermentation. 

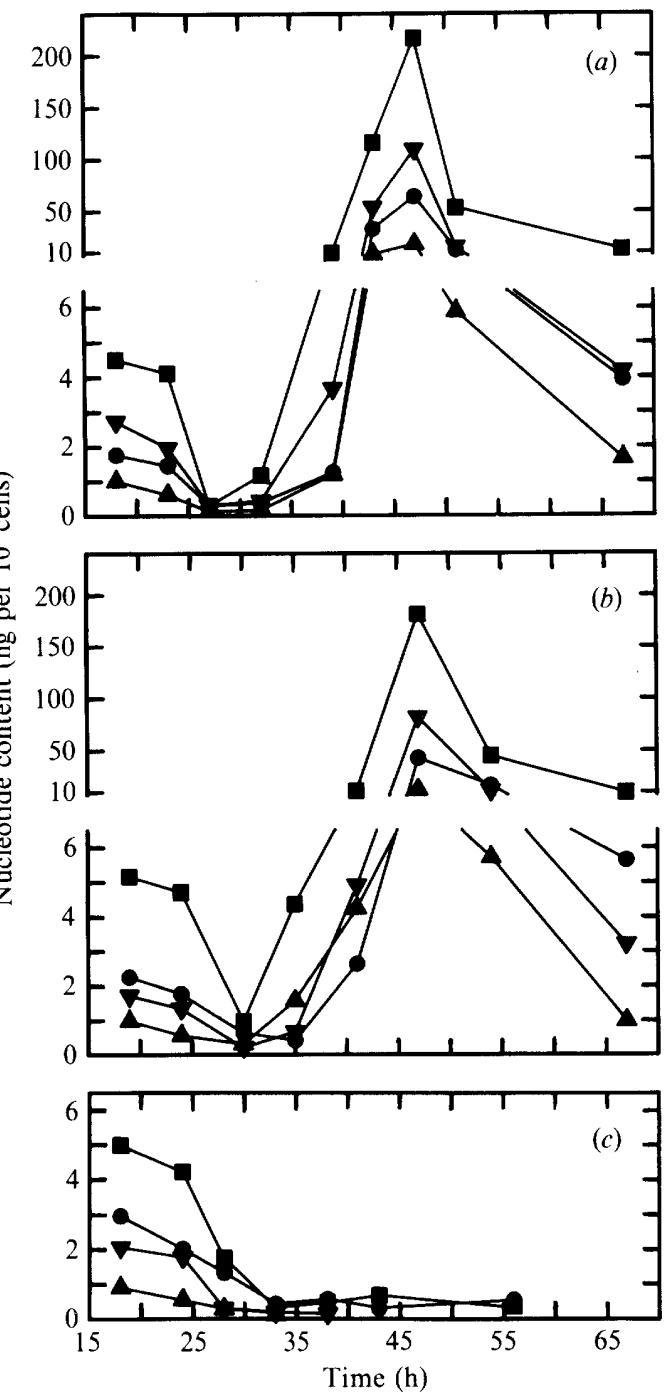

Fig. 2. Nucleoside triphosphate profiles for $C$. acetobutylicum: (a) wild-type strain $\mathrm{P} 262$, (b) the spo-1 mutant, and $(c)$ the $c l s-1$ mutant. $\mathbf{\square}$, ATP; O, GTP; $\mathbf{\Delta}$, CTP; $\nabla$, UTP.

The nucleoside triphosphate profile for the spo-1 mutant (Fig. $2 b$ ) was similar to that for the wild-type P262 strain. ATP was the most abundant nucleoside triphosphate in exponentialphase cells, followed by GTP, UTP and CTP. The spo-1 mutant did not produce forespore septa, but the cells did produce a clostridial stage. The concentrations of all nucleoside triphosphates increased as the percentage of clostridial cells increased, and the peak concentrations coincided with the peak in the percentage of clostridial-stage cells. In contrast to the wild-type strain, the decrease in the nucleoside triphosphate levels after $50 \mathrm{~h}$ was not associated with a decrease in the percentage of clostridial-stage cells. However, the metabolism of the spo- 1 mutant culture slowed down as solvent levels reached their maximum and the glucose concentration decreased.

The $c l s-l$ mutant was unable to produce solvents or to sporulate and did not differentiate into clostridial-stage cells. The nucleotide levels in exponential-phase cells of this mutant (Fig. $2 c$ ) were comparable to those observed in the wild-type strain and the spo- 1 mutant. ATP was the most abundant nucleoside triphosphate, followed by GTP, UTP and CTP. The nucleoside triphosphate levels decreased as the cell number increased and acids were produced, reaching 
Table 1. Percentage sporulation of wild-type C. acetobutylicum P262 with and without the addition of compounds known to alter nucleotide levels

The optimum concentration of these compounds is the concentration that gave the greatest increase in percentage sporulation. The results are representative of two experiments that give similar results.

\section{Compound}

Cordycepin

Decoyinine

Psicofuranine

2-Aminomercaptopurine

6-Mercaptopurine

Caffeine

3-Methylxanthine

Theobromine

Theophylline

6-Azathymine

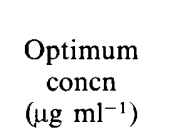

300

100

400

500

400

500

500

200

500

${ }^{100}$

$\overbrace{\begin{array}{c}\text { Without } \\ \text { addition }\end{array}}^{\text {Percentage sporulation }}$

$\begin{array}{ll}33 & 42 \\ 31 & 44 \\ 35 & 53 \\ 39 & 60 \\ 31 & 51 \\ 31 & 60 \\ 35 & 60 \\ 32 & 63 \\ 36 & 57 \\ 36 & 56\end{array}$

almost zero by $35 \mathrm{~h}$. At this time, the cells had stopped dividing and the rate of acid production and glucose consumption had decreased. In contrast to the nucleoside trisphosphate profiles obtained with the wild-type strain and the spo- 1 mutant, the nucleoside trisphosphate levels did not increase during the stationary phase, and after $40 \mathrm{~h}$ UTP and CTP were not detected.

\section{Nucleotide synthesis inhibitors}

Ten compounds which increased the percentage of sporulating cells are shown in Table 1. Cordycepin, decoyinine and psicofuranine are adenosine analogues altered in the ribose moiety (Zain-ul-Abedin et al., 1983). Psicofuranine and decoyinine, which both inhibit GMP synthetase (Freese et al., 1979), were more effective than cordycepin at increasing the percentage of sporulating cells. The mercaptopurines (6-mercaptopurine and 2-aminomercaptopurine) were the next most effective compounds. The methylxanthines caffeine and theobromine provoked the largest increase in percentage sporulation, whereas the other two methylxanthines tested, theophylline and 3-methylxanthine, increased sporulation to a lesser extent. The pyrimidine analogue 6-azathymine also increased the percentage of sporulating cells.

\section{DISCUSSION}

Nutrient shift-down experiments, in which rapidly dividing cells from a rich medium are transferred to a nutrient-depleted medium, have been used to induce sporulation in Bacillus species (Sterlini \& Mandelstam, 1969). However, nutrient depletion does not induce sporulation in C. acetobutylicum P262, which sporulates only under conditions where growth is limited in the presence of excess glucose and ammonia (Long et al., 1984). These studies were therefore carried out in the minimal medium, CAMM, of Long et al. (1983), which provides a sufficiently synchronous developmental cycle to allow for these correlative morphological, physiological and biochemical studies (Woods \& Jones, 1986).

ATP was the most abundant nucleoside triphosphate produced by the wild-type strain and the spo- 1 and $c l s-1$ mutants. An interesting observation was the extremely high concentrations of nucleotides in non-growing clostridial-stage cells of the wild-type strain and the spo-1 mutant, since the fermentation of glucose to solvents yields 2 mol ATP per mol glucose, as compared to approximately $3.3 \mathrm{~mol}$ ATP per mol glucose when acids are produced (Jones \& Woods, 1986). However, the clostridial cells do not appear to elongate or divide and the nucleotides may accumulate as a result of the absence of growth. The high pool values of these energy-rich 
compounds may explain the association of energy-consuming processes such as granulose formation and capsule production with the clostridial stage (Reysenbach et al., 1986).

The units that have been used for reporting nucleoside triphosphate levels in B. subtilis are pmol per $\mathrm{OD}_{600}$ unit (Ochi \& Freese, 1983; Zain-ul-Abedin et al., 1983) or pmol per AM unit (Freese et al., 1981), where one AM unit is the amount of cells that produces an OD $_{600}$ of 1 if suspended in $1 \mathrm{ml}$. Unfortunately, these authors make no reference to cell number, nor do they mention the path length used in their optical density measurements. Therefore, the results presented here, which are based on cell number, are not directly comparable to those above which are based on optical density. With our strain of $C$. acetobutylicum, $10^{8}$ cells $\mathrm{ml}^{-1}$ gives an $\mathrm{OD}_{600}$ of approximately 1 if a $1 \mathrm{~cm}$ path length is used. Therefore if the maximum nucleoside triphosphate concentrations reported by Zain-ul-Abedin et al. (1983) are converted from pmol per $\mathrm{OD}_{600}$ unit to ng per $\mathrm{OD}_{600}$ unit, the values obtained are approximately $375,115,91$ and 41 for ATP, GTP, UTP, and CTP respectively. Our results indicate that the nucleoside triphosphate levels for $1 \times 10^{8}$ C. acetobutylicum cells fall within the same range as reported for 1 $\mathrm{OD}_{600}$ unit of $B$. subtilis cells.

The isolation of various classes and combinations of sporulation, clostridial cell, solvent, granulose and capsule mutants demonstrates that although the individual pathways function independently of each other, the induction of these events appears to be linked by some common regulatory mechanism (Woods \& Jones, 1986). The concentrations of nucleoside triphosphates in the wild-type strain and in the $c l s-1$ and $s p o-1$ mutants decreased during the late-exponential growth phase and reached their lowest levels just prior to the $\mathrm{pH}$ break point. This trough also coincided with a decrease in motility and rate of cell division. In the wild-type strain and the spo- 1 mutant the conversion of cells to the non-dividing clostridial stage and the onset of solvent production coincided with a rapid and very large increase in nucleotide pools. In the $\mathrm{cls}-1$ mutant, which failed to produce solvents or the clostridial stage, no increase in the level of nucleoside triphosphates was detected. The accumulation of high concentrations of nucleoside triphosphates in the spo-l mutant suggests that the increase in these compounds was associated with the conversion of cells to the solvent-producing clostridial stage and was not dependent on spore formation.

A decrease in GTP has been shown to be associated with the initiation of differentiation and sporulation in B. subtilis (Lopez et al., 1979; Freese, 1981; Ochi \& Freese, 1983), Saccharomyces cerevisiae (Freese et al., 1984b), and a streptomycete (Ochi, 1986). A variety of inhibitors which are known to decrease ribonucleotide levels in $B$. subtilis bring about an increase in sporulation (Freese et al., 1979; Ochi \& Freese, 1983; Zain-ul-Abedin et al., 1983). Although there is no direct evidence implicating the low levels of GTP which occur during the late-exponential growth phase in C. acetobutylicum P262 it is possible that a decrease in this or other nucleoside triphosphates plays a role in differentiation of $C$. acetobutylicum. The involvement of these compounds is supported by the increase in sporulation which was obtained in cultures treated with the various inhibitors shown in Table 1 . When these inhibitors were added to stationaryphase cultures just after the $\mathrm{pH}$ break point, little or no increase in sporulation occurred (not shown). The methylxanthines were the most effective compounds in stimulating sporulation, which is consistent with the results of Sacks \& Thompson (1977). One inhibitor which would not be expected to affect purine ribonucleotide levels is the pyrimidine analogue 6-azathymine. This compound also caused an increase in sporulation in $C$. acetobutylicum. Similar results were obtained by Craven \& Blankenship (1982) with Clostridium perfringens.

\section{REFERENCES}

Bagnara, A. S. \& FinCh, L. R. (1972). Quantitative extraction and estimation of intracellular nucleoside triphosphates of Escherichia coli. Analytical Biochemistry 45, 24-34.

Craven, S. E. \& Blankenship, L. C. (1982). Effect of purine derivatives, papaverine hydrochloride, and imidazole on enterotoxin formation by Clostridium perfringens type A. Canadian Journal of Microbiology 28, 851-859.

FREESE, E. (1981). Initiation of bacterial sporulation. In Spores VIII, pp. 1-12. Edited by P. Gerhardt. Washington, DC: American Society for Microbiology.

Freese, E., Lopez, J. M. \& Freese, E. B. (1979). 
Initiation of bacterial and yeast sporulation by partial deprivation of guanine nucleotides. In Regulation of Macromolecular Synthesis by Low Molecular Weight Mediators, pp. 127-143. Edited by D. Richter \& G. Koch. New York: Academic Press.

Freese, E., Lopez, J. M. \& OCHI, K. (1981). Role of guanine nucleotides and of the stringent response to amino acid deprivation in the initiation of bacterial sporulation. In Microbiology-1981, pp. 11-16. Edited by $D$. Schlessinger. Washington, DC: American Society for Microbiology.

Freese, E., Olempska-Beer, Z. \& EisenberG, M. (1984a). Nucleotide composition of cell extracts analysed by full spectrum recording in high pressure liquid chromatography. Journal of Chromatography 284, 125-142.

Freese, E. B., Olempska-Beer, Z., Hartig, A. \& FREESE, E. (1984b). Initiation of meiosis and sporulation of Saccharomyces cerevisiae by sulfur or guanine deprivation. Developmental Biology 102, 438-451.

Hongo, M., Murata, A., Kono, K. \& Kato, F. (1968). Lysogeny and bacterogenicity in strains of Clostridium species. Agricultural and Biological Chemistry 32, 27-33.

JoNES, D. T. \& WoODS, D. R. (1986). Acetone-butanol fermentation revisited. Microbiological Reviews 50, 484-524.

Jones, D. T., van Der Westhuizen, A., Long, S., AlLCOCK, E. R., ReID, S. J. \& WoOdS, D. R. (1982). Solvent production and morphological changes in Clostridium acetobutylicum. Applied and Environmental Microbiology 43, 1434-1439.

Long, S., Jones, D. T. \& Woods, D. R. (1983). Sporulation of Clostridium acetobutylicum P262 in a defined medium. Applied and Environmental Microbiology 45, 1389-1393.

LoNG, S., JoNes, D. T. \& Woods, D. R. (1984). Initiation of solvent production, clostridial stage and endospore formation in Clostridium acetobutylicum P262. Applied Microbiology and Biotechnology 20, 256-261

Lopez, J. M., Marks, C. L. \& Freese, E. (1979). The decrease of guanine nucleotides initiates sporulation of Bacillus subtilis. Biochimica et biophysica acta 587 , 238-252.

MEYER, R.\&WAGNER, K. G. (1985). Determination of nucleotide pools in plant tissue by high performance liquid chromatography. Analytical Biochemistry 148, 269-276.

O'Brien, R. W. \& Morris, J. G. (1971). Oxygen and the growth and metabolism of Clostridium acetobutylicum. Journal of General Microbiology 68, 307-318.

OCHI, K. (1986). A decrease in GTP content is associated with aerial mycelium formation in Streptomyces MA406-A-1. Journal of General Microbiology 132, 299--305.

OCHI, K. \& FreEse, E. (1983). Effect of antibiotics on sporulation caused by the stringent response in Bacillus subtilis. Journal of General Microbiology 129 , 3709-3720.

Olempska-Beer, Z. \& Freese, E. B. (1984). Optimal extraction conditions for high performance liquid chromatographic determination of nucleotides in yeast. Analytical Biochemistry 140, 236-245.

PAYNE, S. M. \& AMES, B. (1982). A procedure for rapid extraction and high pressure liquid chromatographic separation of nucleotides and other small molecules from bacterial cells. Analytical Biochemistry 123, $151-161$.

Reysenbach, A. L., Ravenscroft, N., Long, S., JoNes, D. T. \& Woods, D. R. (1986). Characterization, biosynthesis and regulation of granulose in Clostridium acetobutylicum. Applied and Environmental Microbiology 52, 185-190.

SAcks, L. E. \& ThOMPSON, P. A. (1977). Increased spore yields of Clostridium perfringens in the presence of methylxanthines. Applied and Environmental Microbiology 34, 189-193.

Santangelo, J. D. (1988). A general purpose computer analysis system for chromatographic data. Computer Applications in the Biosciences 4, 275-279.

Sterlini, J. M. \& Mandelstam, J. (1969). Commitment to sporulation in Bacillus subtilis and its relationship to development of actinomycin resistance. Biochemical Journal 113, 29-37.

Woods, D. R. \& Jones, D. T. (1986). Physiological responses of Bacteroides and Clostridium strains to environmental stress factors. Advances in Microbial Physiology 27, 1-64.

Zain-ul-Abedin, Lopez, J. M. \& Freese, E. (1983). Induction of bacterial differentiation by adenineand adenosine-analogs and inhibitors of nucleic acid synthesis. Nucleosides and Nucleotides 2, 257-274. 